\title{
Ações de prevenção e controle do tabagismo no ambiente escolar: Relato de experiência
}

Actions for the prevention and control of tobacco use in the school environment: An experience report

Acciones para la prevención y control del tabaquismo en el entorno escolar: Un relato de experiencia

Raniel Rodrigues Souza

ORCID: https://orcid.org/0000-0002-4953-5853 Universidade do Estado do Pará, Brasil

E-mail: raniel.souza@aluno.uepa.br

Agliely Gomes Pereira

ORCID: https://orcid.org/0000-0002-3995-1677 Universidade do Estado do Pará, Brasil E-mail: agliely.pereira@ aluno.uepa.br

Marcus Vinícius de Arruda Almeida ORCID: https://orcid.org/0000-0002-0238-2992 Universidade do Estado do Pará, Brasil E-mail: marcus.almeida@aluno.uepa.br

Yasmin Luana Andrade Rodrigues ORCID: https://orcid.org/0000-0003-2950-0730 Universidade do Estado do Pará, Brasil

E-mail: yasmim.rodrigues@aluno.uepa.br

Orácio Carvalho Ribeiro Júnior ORCID: https://orcid.org/0000-0003-3212-9936 Universidade do Estado do Pará, Brasil E-mail: oracio.junior@uepa.br

Kaio Vinícius Paiva Albarado ORCID: https://orcid.org/0000-0002-0687-7124

Universidade Federal do Oeste do Pará, Brasil E-mail: kaioalbarado@gmail.com

Semírames Cartonilho de Souza Ramos ORCID: https://orcid.org/0000-0001-8370-5994

Universidade Federal da Paraíba, Brasil

E-mail: semiramesramosmoga@gmail.com

Tatiane Silva de Araújo ORCID: https://orcid.org/0000-0003-1657-5565

Universidade Federal do Amazonas, Brasil

E-mail: tatiane-araujo@live.com

Tayane Moura Martins ORCID: https://orcid.org/0000-0003-3236-8574 Universidade Federal do Pará, Brasil

E-mail: tayane_m.martins1@ @otmail.com

Rosane Silva dos Santos ORCID: https://orcid.org/0000-0002-2872-3505 Universidade do Estado do Pará, Brasil E-mail: rosane.ssantos@hotmail.com

Suzana Maria da Silva Ferreira ORCID: https://orcid.org/0000-0001-9774-4820 Universidade Federal de Roraima, Brasil E-mail: suzana-ferreira@live.com

Aline Verçosa de Figueiredo ORCID: https://orcid.org/0000-0001-8752-6432 Universidade do Estado do Pará, Brasil

E-mail: v.alinne@yahoo.com.br

William Cardoso da Cunha

ORCID: https://orcid.org/0000-0001-7660-0879 Hospital Geral de Altamira São Rafael, Brasil

E-mail: enferobwilliamcunha@gmail.com 


\begin{abstract}
Resumo
Objetivo: Relatar as ações educativas, realizadas com escolares adolescentes, sobre os efeitos nocivos do tabagismo e a importância de não iniciar e, quando for o caso, de parar com o hábito de fumar. Método: Trata-se de um relato de experiência das atividades integradas em saúde, realizadas por estudantes do curso de enfermagem de uma instituição de ensino superior pública situada na Região do Xingu, em novembro de 2019. As ações educativas foram balizadas pela metodologia da problematização, tendo como operar prático o Arco de Maguerez. Resultados: Após o contato inicial com o público-alvo para verificar seus conhecimentos prévios e carências sobre a temática, os acadêmicos elaboraram estratégias para promover o momento educativo que foi operacionalizado por meio de uma vídeo-aula projetada para os escolares sobre os componentes do cigarro. Além disso, foi realizado um quiz por meio do aplicativo plickers no qual os alunos puderam interagir com os acadêmicos, esclarecendo suas dúvidas sobre a temática abordada. Após isso, foram realizadas duas demonstrações práticas em ambiente aberto, com simuladores artesanais, desenvolvidos pelos próprios acadêmicos, sobre os efeitos deletérios do uso de cigarros para o pulmão. Todas as atividades tiveram anuência da gestão escolar e foram desenvolvidas sob supervisão de um docente. Considerações Finais: As atividades educativas foram de extrema importância, pois delinearam-se a partir das necessidades de conhecimentos dos escolares, sanando suas dúvidas sobre a temática. Para os graduandos, a experiência permitiu o desenvolvimento do currículo integrado do curso de enfermagem, além de qualificar o processo de formação sobre a temática.
\end{abstract}

Palavras-chave: Prevenção do hábito de fumar; Educação em saúde; Adolescência.

\begin{abstract}
Objective: To report the educational activities carried out with adolescent students about the harmful effects of smoking and the importance of not starting and, when appropriate, stopping the habit of smoking. Method: This is an experience report of integrated activities in health, carried out by nursing students of a public higher education institution located in the Xingu Region, in November 2019. The educational actions were guided by the problematization methodology, with the practical operation of the Arco de Maguerez. Results: After initial contact with the target audience to verify their prior knowledge and needs on the subject, the students developed strategies to promote the educational moment that was operationalized through a video lesson designed for students about the components of cigarettes. In addition, a quiz was conducted through the plickers application where students could interact with academics, clarifying their doubts about the topic addressed. After that, two practical demonstrations were carried out in an open environment, with handcrafted simulators, developed by the students themselves, on the harmful effects of cigarette use on the lung. All activities had the consent of the school management and were developed under the supervision of a teacher. Final Considerations: The educational activities were extremely important, as they were delineated from the students' knowledge needs, solving their doubts about the theme. For undergraduates, the experience allowed the development of the integrated curriculum of the nursing course, in addition to qualifying the training process on the subject.
\end{abstract}

Keywords: Smoking prevention; Health education; Adolescence.

\title{
Resumen
}

Objetivo: Informar las actividades educativas realizadas con estudiantes adolescentes sobre los efectos nocivos del tabaquismo y la importancia de no iniciar y, en su caso, detener el hábito de fumar. Método: Se trata de un relato de experiencia de actividades integradas en salud, realizadas por estudiantes de enfermería de una institución pública de educación superior ubicada en la Región Xingu, en noviembre de 2019. Las acciones educativas fueron guiadas por la metodología de problematización, con el funcionamiento práctico de la Arco de Maguerez. Resultados: Luego del contacto inicial con el público objetivo para verificar sus conocimientos y necesidades previas sobre el tema, los estudiantes desarrollaron estrategias para promover el momento educativo que se operacionalizó a través de una videolección diseñada para los estudiantes sobre los componentes de los cigarrillos. Además, se realizó un cuestionario utilizando la aplicación plickers donde los estudiantes pudieron interactuar con académicos, aclarando sus dudas sobre el tema abordado. Posteriormente, se realizaron dos demostraciones prácticas en un ambiente abierto, con simuladores artesanales, desarrollados por los propios alumnos, sobre los efectos nocivos del uso de cigarrillos en el pulmón. Todas las actividades contaron con el consentimiento de la dirección de la escuela y se desarrollaron bajo la supervisión de un docente. Consideraciones finales: Las actividades educativas fueron de suma importancia, ya que se delinearon a partir de las necesidades de conocimiento de los estudiantes, resolviendo sus dudas sobre el tema. Para los estudiantes de pregrado, la experiencia permitió el desarrollo del plan de estudios integrado del curso de enfermería, además de calificar el proceso de formación en la materia.

Palabras clave: Prevención del tabaquismo; Educación para la salud; Adolescencia.

\section{Introdução}

Tabagismo é o nome dado ao uso do tabaco (Nicotiana tabacum). É identificado como uma doença crônica, causando dependência a nicotina, substância presente nos produtos a base do tabaco. Parar de fumar é o principal motivo evitável para o progresso de doenças e mortes no mundo (Raja et al., 2016; Samet, 2013; World Health Organization, 2013). De modo específico 
o tabaco atinge negativamente o transporte mucociliar, ferramenta de defesa do sistema respiratório, além de danificar o exercício pulmonar por outros mecanismos (Addicott et al., 2014; Cohen, 2009; Uzeloto et al., 2017). As práticas de consumo e as externalidades do tabaco estão diretamente ligados a 16 tipos distintos de câncer, sendo o câncer de pulmão o principal deles, e ainda atua como fator de risco para várias doenças crônicas e respiratórias (Borges et al., 2020).

O uso contínuo do tabaco ocasiona mudanças negativas para o corpo, causando déficits no funcionamento da respiração, além de debilitar várias funções do organismo (Stabbert et al.,2017; Olivetti, 2013). Conforme Oliveira-Campos et al. (2018) o uso de tabaco pela população jovem aumenta significativamente os problemas de saúde ao longo da infância e da adolescência. Dessa forma, a fase da adolescência pode desencadear situações que atendam a atenção dos serviços públicos focados em vulnerabilidades, como a gravidez não planejada, uso de tabaco, abuso de álcool dentre outras drogas (Ferreira et al., 2016).

Em um estudo realizado no Peru, descobriu-se que a maior parte dos adolescentes começaram a fazer o uso do tabaco aos 14 anos, motivados pelos amigos, pela curiosidade e, sem o conhecimento dos pais (Valladares-Garrido et al., 2017). No Brasil foi verificado pela Pesquisa Nacional de Saúde Escolar (PeNSE) que 30\% dos adolescentes entre 13 e 15 anos, iniciaram o processo do uso do tabaco antes dos 12 anos. Ademais, é retratado que os costumes adquiridos nesse estágio da vida são mantidos até a fase adulta e, no geral, não sofrem modificações, mesmo esses jovens sendo conhecedores sobre os riscos que o tabaco causa para a saúde, os seus hábitos contrariam esse conhecimento. Geralmente, é na fase de passagem do ensino médio para o ensino superior que os jovens iniciam com o consumo do tabaco e estabilizam a atitude de fumar (Urrutia-Pereira, et al., 2017).

No Brasil, o Programa Nacional de Controle do Tabagismo (PNCT) atua com a rede de tratamento gratuito no Sistema Único de Saúde (SUS) para promover a cessação do hábito de fumar através de práticas ampliadas e intersetoriais que contribuam para o objetivo macro do programa (Brasil, 2020). O desempenho de políticas públicas deve ser eficaz para impedir o início precoce do uso do tabaco entre adolescentes, analisando não apenas as características individuais ou familiares, mas também as características do bairro e a escola em que estudam (Kim \& Chun, 2018). No estudo de Boing et al. (2019) foi observado que a prevalência de fumar é maior entre adolescentes que não completaram o ensino fundamental, enquanto o uso de outros tipos de tabaco foi maior entre os filhos de mães com maior escolaridade.

Conforme Moura et al. (2018) as causas para a prática do tabagismo na adolescência são multifatoriais e estão relacionadas ao âmbito em que o indivíduo está inserido, incluindo-se o ambiente escolar, assim como fatores econômicos e sociais que também influenciam nesse hábito. De acordo com Freitas, Martins e Espinosa (2019), a chance de um adolescente experimentar o álcool é mais provável para o sexo feminino, enquanto o tabaco é mais frequente para o sexo masculino, sendo cada vez mais comum em idades prematuras. Contudo, a escola é um espaço essencial na prevenção do tabagismo e de outras drogas, devido ser um ambiente utilizado para o desenvolvimento de ações em educação de saúde e com isso, torna-se um lugar favorável ao desenvolvimento de uma visão crítica dos jovens, que acarreta na construção de responsabilidade social e da saúde (Porto et al., 2018).

Assim, práticas educativas nos ambientes escolares tem se tornado cada vez mais comuns, porém, com diferentes abordagens metodológicas, que possam apreender a atenção do público-alvo e, ao mesmo tempo, esclarecedoras sobre os temas relacionados ao processo saúde-doença, neste caso, o tabagismo. Diante do exposto, o presente trabalho tem como objetivo relatar as ações educativas, realizadas com escolares adolescentes, sobre os efeitos nocivos do tabagismo e a importância de não iniciar e, quando for o caso, de parar com o hábito de fumar.

\section{Metodologia}

Trata-se de um estudo de natureza descritiva com abordagem qualitativa do tipo relato de experiência, a partir de uma determinada experiência vivenciada na prática das Atividades Integradas em Saúde (AIS) com a temática “Ações de Prevenção 
e Controle do Tabagismo no Ambiente Escolar", realizada junto à uma comunidade escolar da rede pública de ensino fundamental na zona urbana na cidade de Altamira/Pa.

A ação foi desenvolvida em um encontro por acadêmicos do primeiro semestre do curso de Enfermagem da Universidade do Estado do Pará, Campus Altamira, com a duração de 90 minutos, perante a supervisão de duas docentes enfermeiras da universidade.

O trabalho foi embasado na metodologia da problematização com o Arco de Maguerez, cujas etapas ajudam a identificar o problema e a escolha de intervenções para a mudança da realidade, dividindo-se em cinco etapas: observação da realidade, levantamento de pontos-chave, teorização, levantamento das hipóteses de solução e retorno à realidade para a aplicação da ação (Berbel, 2011).

A primeira etapa do arco, é a observação da realidade que consiste no envolvimento ativo dos sujeitos (os alunos) para uma atenção crítica da realidade, então é analisada uma primeira leitura sobre o tema a ser implantado ou inserido na vida real. É hora dos sujeitos envolvidos olharem de perto a realidade, identificando aspectos que necessitam ser trabalhados, desenvolvidos ou melhorados (Berbel, 1998; Bordenave, 2004).

A complexidade na preparação do problema torna os alunos mais críticos, contextualizá-los em busca de uma solução. Com base em críticas, as pessoas têm a tarefa de formular pontos para facilitar a compreensão e, posteriormente, interferir no contexto em que surgem o problema, esses pontos são chamados de pontos chaves que são elaborados na segunda etapa do arco (Berbel, 1998). Em seguida é realizada a teorização do problema, onde procura-se compreender os pontos chaves estabelecidos anteriormente, para uma possível resolução do problema (Berbel, 1998).

Posteriormente, a quarta fase do arco de Charles Maguerez consiste na preparação de alternativas viáveis para resolver o confronto entre a teoria e a realidade de forma crítica e criativa (Reibnitz, 2006). A quinta e última etapa consiste no "retorno à realidade para a aplicação da ação" na qual os alunos devem aplicar decisões e respostas encontradas para resolver os problemas listados nas primeiras quatro etapas. A prática no campo procura soluções e representa uma transformação para a realidade observada, o que contribui para o conhecimento e ajuda com o processo de ensino (Berbel, 1998).

Para a execução do plano, foi necessário a utilização de tecnologias como projetor, celular, notebook e aparelho de som. As atividades ocorreram dentro de uma escola no município em novembro de 2019 , com uma turma do nono ano da qual participaram 27 alunos de ambos os sexos, com idades entre 14 e 16 anos.

\section{Resultados}

As AIS tiveram a finalidade de esclarecer aos alunos da escola acerca do tabagismo e suas consequências, para levá-los a uma reflexão crítica sobre a temática, diminuindo dessa forma a influência sobre esses jovens para o consumo. Com isso, o desenvolvimento do plano para efetivar essa ação foi apresentado para os jovens, levando informação de forma objetiva e lúdica para os mesmos.

A partir das propostas do arco de Maguerez, na primeira etapa, foi a realizado uma visita na escola para a observação da realidade, com o objetivo de perceber as situações-problema que a envolvem, expressando desse modo percepções pessoais e selecionando uma situação a ser problematizada. Dessa forma, os discentes perceberam que no entorno da escola tinha uma grande quantidade de bituca de cigarros, considerando esse fato como ponto chave e de maior relevância em se tratando de uma escola de ensino fundamental.

Posteriormente, na segunda etapa do arco, os docentes e discentes se reuniram para destacarem o que foi observado durante a visitação, o que é verdadeiramente importante, procurando identificar os pontos-chave do problema em questão, ocasionando a busca pela elaboração de possibilidades de solução. Diante disso, foi concluído a necessidade de intervenções com a proposta de transferir conhecimentos sobre a problemática, para a mudança da realidade da escola visitada. 
A teorização foi realizada através de leituras dos artigos científicos localizados em bancos de dados digitais que serviram de alicerce para o embasamento da ação. No andamento do arco, na etapa de levantamento das hipóteses de solução, foi decidido a elaboração de uma ação educativa vinculada a temática “AÇÕES DE PREVENÇÃO E CONTROLE DO TABAGISMO NO AMBIENTE ESCOLAR”, no intuito de contribuir com a redução do tabagismo entre os adolescentes da escola.

Por fim, o retorno à realidade para a aplicação da ação, foi caracterizada pela prática das alternativas de solução que o grupo encontrou como prováveis, essas atividades foram planejadas e, subsequentemente, retornando à realidade observada na primeira etapa. A ação seguiu da seguinte forma: bate papo inicial acerca da patologia, apresentação do tema em vídeo aula criada pelos docentes da instituição, aplicação de um quiz com o uso do aplicativo de celular, aplicação de duas experiências como forma de conscientizar os alunos quanto aos riscos de fumar e, no fim, foi aberto para eventuais dúvidas.

No primeiro contato, pôde ser observado nos alunos carência de informações a respeito do tabagismo, possibilitando curiosidade e aprendizado sobre o tema, foi notado também que alguns alunos possuíam fumantes em seu grupo familiar. De acordo com Raimundo (2019), a família é responsável pelo primeiro vínculo e hábitos entre os indivíduos que podem durar por toda a vida, estando diretamente relacionada com a influência para a inicialização e desenvolvimento ao tabagismo.

Além disso, é válido ressaltar que os alunos compreendiam parcialmente o tema abordado, porém ao longo da apresentação do vídeo aula foi perceptível que os escolares começaram a compreender o assunto. Após a vídeo aula, foram realizadas perguntas relacionadas ao que foi apresentado em um quiz por meio de um aplicativo de celular (Plickers), onde os alunos puderam interagir com os acadêmicos, esclarecendo suas dúvidas sobre a temática abordada.

Logo após, foi realizada uma demonstração, na qual foi utilizado um fumante fictício, que foi confeccionado pelos acadêmicos com o uso de uma caixa de sapato, algodão, um pote de plástico e uma seringa. Possuía um sistema no qual o cigarro era introduzido em um buraco na caixa, o algodão era preso na tampa do pote, no momento que ocorria a tragada do cigarro com a ajuda da seringa, o algodão ficava amarelado, sendo possível visualizar como permanecia retido no filtro as substâncias do cigarro que são causadoras de câncer e várias doenças relacionadas ao tabaco, provocando um impacto para todos os presentes durante a demonstração.

Em seguida, na segunda demonstração prática, foi utilizado uma garrafa com água que representava o pulmão. Nela havia um furo na tampa para que fosse encaixado o cigarro e um furo na parte inferior, que se encontrava tampado. No momento da demonstração tiramos o tampão do furo inferior e a água começou a esvaziar aos poucos e, diante disso, ia tragando o cigarro, sendo possível visualizar o interior da garrafa ficando completamente cheio de fumaça. Posteriormente, com a garrafa sem água foi retirado a tampa da garrafa juntamente com a bituca do cigarro e foi amarrado um guardanapo no seu lugar para que fosse possível visualizar o que ocorria no momento em que a fumaça no seu interior foi expulsa, onde foi possível observar o guardanapo totalmente amarelado indicando as substâncias tóxicas presente no tabaco. Diante disso, os discentes explicaram como fica o pulmão de um fumante com a inalação da fumaça, o mal que ela causa e que na traqueia paralisa temporariamente os cílios que limpam o muco e outras partículas invasoras do sistema respiratório ao mesmo tempo em que a nicotina passa para a corrente sanguínea. Dessa maneira, foi demonstrado na prática os efeitos nocivos do tabaco, levando intervenções conjuntas entre universidade e instituições de ensino.

O projeto resultou na expansão de conhecimento sobre o Tabagismo, aumentando a análise crítica dos alunos. O mesmo fez com que seja possível diminuir cada vez mais os índices de tabagismo nos jovens, um caso de saúde pública que deve ser cessado o mais cedo possível. Com isso, percebe-se a importância de trabalhar em instituições de ensino levando ações que promovam uma interface com a saúde. Outrossim, permite vivências e benefícios para todos os envolvidos, possibilitando mudanças nas ações de saúde na comunidade. 


\section{Discussão}

O tabaco está diretamente ligado a óbitos por Doença Pulmonar Obstrutiva Crônica (DPOC) - enfisema e bronquite, diversos tipos de cânceres (boca, pulmão, laringe, esôfago, faringe, rim, bexiga, pâncreas, estômago, colo de útero e fígado), e, ainda, por doenças cerebrovasculares e coronarianas (Amarante, 2018).

A nicotina presente no cigarro é uma droga, e como tal age no sistema nervoso central causando efeitos nocivos diversos. Além disso, foram identificadas mais de 7.000 substâncias presentes na fumaça do cigarro, sendo que no mínimo 69 delas são consideradas cancerígenas (Brasil, 2020). Ademais, pesquisas reveladas por Raimundo (2019), comprovam que a fumaça do cigarro é responsável pela instigação da apoptose nas células ciliadas do sistema respiratório e pela diminuição da disponibilidade celular.

O uso do tabaco é considerado uma epidemia e um problema à saúde pública, anualmente é responsável por mais de 80 milhões de mortes no mundo, entre essas mortes mais de 7 milhões são ocasionadas pelo uso direto do tabaco e mais de 1,2 milhões pelo uso indireto, ou seja, essa parcela é exposta a fumaça de forma indireta, reconhecidos como fumantes passivos (Brasil, 2020). Hodiernamente no Brasil, diariamente morrem 428 pessoas devido a dependência a nicotina, e 156.215 mortes anuais poderiam ser evitadas. Os maiores índices são ligados a doença cardíaca, câncer, DPOC, Acidente Vascular Cerebral (AVC) e pneumonia (Pinto et al. 2019).

De acordo com Barbalho et al. (2021), é identificado uma assistência em saúde insuficiente em relação a sensibilização dos usuários de cigarro, uma vez que os danos ocasionados à saúde são preocupantes, porém o uso de metodologias ativas é indispensável para reverter tal situação, visto que os indivíduos possam se integrar de forma fácil no processo de escape do tabagismo.

Estudos relatam que as escolas públicas têm maiores índices para a experimentação do tabaco e consequentemente o seu uso nos últimos 30 dias são mais recorrentes nessas instituições (Brasil, 2020). Conforme Ribeiro et al. (2017) o grande problema é que as drogas podem causar dependência física e psicológica, bem como outros danos, como acidentes, violência, suicídio e transmissão de doenças.

Os resultados apresentados neste relato corroboram com o estudo de Rios e Freire (2020) no qual foi analisado 3.034 estudantes adolescentes, destes, 824 (27,2\%) afirmaram ter usado cigarro pelo menos uma única vez, tendo 241 (7,9\%) fumado nos últimos 30 dias e $50(1,7 \%)$ fumam todos os dias. Nesse sentido, é evidenciado que o ambiente escolar deve ser um local que proporcione aprendizado seguro e desenvolvimento integral de crianças e adolescentes, auxiliando nas situações que corresponda a riscos à saúde física e psicológica, assim como deve ser um agente de saúde, adotando ações de promoção e orientação à saúde (Porto et al., 2018). Além disso, o processo de educação em saúde é um dos esquemas usados para promoção em saúde que possui o intuito de corroborar com a construção do pensamento crítico, a fim de possibilitar estratégias para solucionar problemas e não apenas seguir recomendações ou normas, bem como oferecer reforço para o indivíduo adotar novas condutas de saúde (Ferreira, 2006).

No decorrer da história, o profissional de enfermagem se inseriu como ator principal no processo de educação em saúde, visto que o ato de cuidar é inseparável ao diálogo e à educação. Esse processo exige constantemente do profissional a consciência crítica da importância como educador. Ao realizar educação em saúde, o enfermeiro não está apenas trazendo o conhecimento, mas também, elaborando uma ferramenta que desenvolve o senso crítico, tornando sujeito ativo no processo de promoção de saúde, proporcionando tomadas de decisões mais precisas para exercer sua cidadania (Ramos et al. 2021).

Diante disso, a prática educativa que foi realizada na escola contribuiu ativamente para os saberes daqueles alunos, visto que essas práticas levam o acadêmico a atuar diretamente com a comunidade tendo relação direta com a sua profissão (enfermagem). 


\section{Considerações Finais}

A experiência de levar a AIS aos discentes de uma escola de ensino fundamental com uma temática relacionada à saúde pública viabilizou a aproximação e conhecimentos dos jovens sobre o tabagismo, possibilitando mais interação dos alunos com a temática. Nesse sentido, foi possível analisar a carência de informações acerca do tema e os impasses existentes na prática de aplicar o projeto.

Tal ação contribuiu para a construção e aprimoramento do saber-fazer da enfermagem e no emocionante processo de trabalho, que se caracteriza também pelo ensinar-aprender, enriquecendo os aplicadores do projeto e os alunos, pois aponta princípios para uma educação acerca do tema, que pode ser levado para fora do campo escolar, propagando uma produção de cuidado em saúde.

Cabe ressaltar que estudos dessa temática envolvendo a participação das universidades públicas da região amazônica, são pouco propagados no meio acadêmico. Por isso, deu-se ênfase sobre realização desse estudo por uma das mais conceituadas e respeitadas instituições de ensino da região norte do Brasil. Levar o conhecimento técnico-científico para a população, transcendendo as paredes dos centros universitários, deve ser realizado constantemente, fazendo valer o tripé acadêmico.

\section{Referências}

Addicott, M. A., Froeliger, B., Kozink, R. V., Van Wert, D. M., Westman, E. C., Rose, J. E., \& McClernon, F. J. (2014). Nicotine and non-nicotine smoking factors differentially modulate craving, withdrawal and cerebral blood flow as measured with arterial spin labeling. Neuropsychopharmacology, 39(12), 27502759. https://www.nature.com/articles/npp2014108.

Amarante, S. (2018). O cigarro e os seus malefícios. Fiocruz.

Barbalho, I. L. A., Leandro, G. B., da Silva, R. D. J. R., de Lima, M. A. G., \& Fernandes, M. C. (2021). Ações educativas como possibilidade no repensar do homem cigano frente ao consumo do cigarro. Revista Enfermagem Atual In Derme, 95(33). https://doi.org/10.31011/reaid-2021-v.95-n.33-art.873.

Berbel, N. A. N. (1998). A problematização e a aprendizagem baseada em problemas: diferentes termos ou diferentes caminhos? Interface-Comunicação, Saúde, Educação, 2(2), 139-154. https://doi.org/10.1590/S1414-32831998000100008.

Berbel, N. A. N. (2011). As metodologias ativas e a promoção da autonomia de estudantes. Semina: Ciências Sociais e Humanas, 32(1), 25-40. http://dx.doi.org/10.5433/1679-0383.2011v32n1p25.

Bordenave, J. D. (2004). Estratégias de ensino-aprendizagem. Vozes.

Borges, L. C., Menezes, H. Z. D., \& Souza, I. M. L. D. (2020). Dilemas na implementação da convenção-quadro para o controle do tabaco da organização mundial da saúde. Cadernos de Saúde Pública, 36(2), 1-15. 10.1590/0102-311X00136919.

Boing, A. C., Boing, A. F., \& Subramanian, S. V. (2019). Association of violence in schools' vicinity and smoking in schools' premises with tobacco use among Brazilian adolescents. Cadernos de Saúde Pública, 35(12), 1-13. 10.1590/0102-311X00057919.

Cohen, N. A., Zhang, S., Sharp, D. B., Tamashiro, E., Chen, B., Sorscher, E. J., \& Woodworth, B. A. (2009). Cigarette smoke condensate inhibits transepithelial chloride transport and ciliary beat frequency. Laryngoscope, 119(11), 2269-2274. https://doi.org/10.1002/lary.20223

Ferreira, M. D. A. (2006). A educação em saúde na adolescência: grupos de discussão como estratégia de pesquisa e cuidado-educação. Texto \& ContextoEnfermagem, 15(2), 205-211. https://doi.org/10.1590/S0104-07072006000200003.

Ferreira, C. P. D. S., Marques, J. F., Rozendo, C. A., Ferreira, C. B., Pinto, L. M. T. R., \& Ferreira, A. D. S. (2016). Educational strategies for health education with teens: a review integrative. Revista de Pesquisa: Cuidado é Fundamental Online, 8(2), 4197-4211. https://doi.org/10.9789/2175-5361.2016.v8i2.41974211 .

Freitas, E. A. D. O., Martins, M. S. A. S., \& Espinosa, M. M. (2019). Experimentação do álcool e tabaco entre adolescentes da região Centro-Oeste/Brasil. Ciência \& Saúde Coletiva, 24(4), 1347-1357. https://doi.org/10.1590/1413-81232018244.15582017.

Instituto Nacional de Câncer. (2020). Programa nacional de controle do tabagismo. Rio de Janeiro.

Kim, H. H. S., \& Chun, J. (2018). Analyzing multilevel factors underlying adolescent smoking behaviors: the roles of friendship network, family relations, and school environment. Journal of School Health, 88(6), 434-443. https://doi.org/10.1111/josh.12630

Moura, L. R. D., Torres, L. M., Cadete, M. M. M., \& Cunha, C. D. F. (2018). Fatores associados aos comportamentos de risco à saúde entre adolescentes brasileiros: uma revisão integrativa. Revista da Escola de Enfermagem da USP, 52.1-11. https://doi.org/10.1590/S1980-220X2017020403304.

Oliveira-Campos, M., Oliveira, M. M. D., Silva, S. U. D., Santos, M. A. S., Barufaldi, L. A., Oliveira, P. P. V. D., Andrade, S. C. A., Andreazzi, M. A. R., Moura, L., Malta, D. C., \& Souza, M. D. F. M. D. (2018). Risk and protection factors for chronic noncommunicable diseases in adolescents in Brazilian capitals. Revista Brasileira de Epidemiologia, 21(1), 1-15. https://doi.org/10.1590/1980-549720180002.supl.1. 
Research, Society and Development, v. 10, n. 8, e3910816867, 2021

(CC BY 4.0) | ISSN 2525-3409 | DOI: http://dx.doi.org/10.33448/rsd-v10i8.16867

Olivetti, R. F. (2013) O tabagismo e suas consequências: uma abordagem sobre a importância da adoção de hábitos saudáveis [Monografia, Universidade Tecnológica Federal do Paraná].

Pinto, M., Bardach, A., Palacios, A., Biz, A., Alcaraz, A., Rodriguez, B., \& Pichon-Riviere, A. (2019). Carga do tabagismo no Brasil e benefício potencial do aumento de impostos sobre os cigarros para a economia e para a redução de mortes e adoecimento. Cadernos de Saúde Pública, 35(8),118. https://doi.org/10.1590/0102-311X00129118.

Porto, D. R. M. (2018). Prevenção do tabagismo e o papel das escolas: um estudo exploratório em uma escola pública. http://dspace.unipampa.edu.br:8080/bitstream/riu/3206/1/Debora\%20Ribeiro\%20 Maciel\%20Porto\%20-\%202018.pdf

Porto, D. R. M., Cappellari, G., Silva, M. L., \& Roncato, P. E. S. (2018). Smoking prevention and the role of schools: a case study. Brazilian Journal of Development, 4(7), 4103-4127. https://www.brazilianjournals.com/index.php/BRJD/article/view/470/407.

Raimundo, D. D. M. P., \& Multiprofissional, R. (2019). A Influência do Meio Social para o Tabagismo. https://docs.bvsalud.org/biblioref/2020/12/1141248/raimundo-daiana-de-matos-ponte-a-influencia-do-meio-social-par_NbXhq1T.pdf

Raja, M., Garg, A., Yadav, P., Jha, K., \& Handa, S. (2016). Diagnostic methods for detection of cotinine level in tobacco users: a review. Journal of Clinic and Diagnostic Research, 10(3), 04-06. 10.7860/JCDR/2016/17360.7423.

Ramos, M., Maciel, M. A., Santos, M. T. F., de Moura Ferreira, M. C., Giuliani, C. D., \& de Barros Junqueira, M. A. (2021). Abordagem do tabagismo nos grupos de sala de espera como estratégia de promoção à saúde. Brazilian Journal of Health Review, 4(2), 5476-5488. 10.34119/bjhrv4n2-118.

Reibnitz, K. S., \& do Prado, M. L. (2006). Inovação e educação em enfermagem. Cidade Futura.

Ribeiro, F. D. O., Ribeiro, G. A. S., \& Willenshofer, I. (2017). A judicialização de medicamentos oncológicos no Estado de São Paulo [Dissertação de Mestrado, Fundação Getúlio Vargas].

Rios, L. E., \& Freire, M. D. C. M. (2020). Opinião de escolares adolescentes fumantes sobre aconselhamento e tratamento para cessação do tabagismo em serviços de saúde: estudo transversal, Goiás, 2018. Epidemiologia e Serviços de Saúde, 29(4), https://doi.org/10.5123/S1679-49742020000400001.

Samet, J. M. (2013). Tobacco smoking: the leading cause of preventable disease worldwide. Thoracic Surgery Clinics, 23(2), 103112. 10.1016/j.thorsurg.2013.01.009.

Stabbert, R., Dempsey, R., Diekmann, J., Euchenhofer, C., Hagemeister, T., Haussmann, H. J., Knorr, A., Mueller, B. P., Pospisil, P., Reininghaus, W., Roemer, E., Tewes, F. J., \& Veltel, D. J. (2017). Studies on the contributions of smoke constituents, individually and in mixtures, in a range of in vitro bioactivity assays. Toxicol In Vitro, 42, 222-246. https://doi.org/10.1016/j.tiv.2017.04.003.

Urrutia-Pereira, M., Oliano, V. J., Aranda, C. S., Mallol, J., \& Solé, D. (2017). Prevalence and factors associated with smoking among adolescents. Jornal de Pediatria, 93(3), 230-237. https://doi.org/10.1016/j.jped.2016.07.003.

Uzeloto, J. S., Ramos, D., Freire, A. P. C.F., Christofaro, D. G. D., \& Ramos, E. M. C. (2018). Nasal mucociliary transportability of male and female smokers. Brazilian Journal of Otorhinolaryngology, 84(3), 311-317. https://doi.org/10.1016/j.bjorl.2017.03.006.

Valladares-Garrido, M. J., Mariñas-Miranda, W., Velasco-Ferreyra, M. P., \& Mejia, C. R. (2017). Socioeducational factors associated with tobacco use in secondary education students in a rural area of northern Peru. Pediatría Atención Primaria, 19(74), 53-58. https://scielo.isciii.es/pdf/pap/v19n74/en_1139-7632pap-19-74-00053.pdf.

World Health Organization. (2013). WHO report on the global tobacco epidemic, 2013: enforcing bans on tobacco advertising, promotion and sponsorship. World Health Organization. https://apps.who.int/iris/bitstream/handle/10665/85381/WHO_NMH_PND_13.2_eng.pdf. 